\title{
A NOTE ON MINIMAL MODULAR SYMBOLS
}

\author{
AVNER ASH ${ }^{1}$
}

\begin{abstract}
For any arithmetic group, a set of geometrically-defined cohomology classes is constructed which spans the cohomology of the group with rational coefficients in the highest nonvanishing dimension thereof.
\end{abstract}

This note gives a geometrical spanning set $S$ for the cohomology of an arithmetic group in the highest nonvanishing dimension. To do this, I generalize the first three sections of [1]. Unfortunately, I know of no way to generalize the algorithm in $\S 4$ of [1]. This means that while the $S$ I construct is infinite, and although a priori the cohomology is finite dimensional, I cannot identify a finite generating set inside $S$, except for the cases covered in $[\mathbf{1}] .^{1}$

Let $G$ be a semisimple algebraic group defined over $\mathbf{Q}, K$ a maximal compact subgroup of $G(\mathbf{R}), X=G(\mathbf{R}) / K$, and $\Gamma$ an arithmetic subgroup of $G(\mathbf{Q})$. The action of $G(\mathbf{R})$, and hence of $\Gamma$, on $X$ is on the left. It is well known that $H^{*}(\Gamma, \mathbf{Q}) \simeq$ $H^{*}(X / \Gamma, \mathbf{Q})$, and by $[\mathbf{2}]$ that $H^{*}(\Gamma, \mathbf{Q})=0$ for ${ }^{*}>N$, where

$$
N=\operatorname{dim} X-\operatorname{rank}_{\mathbf{Q}}(G) .
$$

To construct $S \subset H^{N}(X / \Gamma, \mathbf{Q})$ we proceed as follows: Let $T$ be a maximal $\mathbf{Q}$ split torus of $G$. Without loss of generality, we may assume that $T$ is stable under the Cartan involution of $G$ corresponding to $K$. Set $A=T(\mathbf{R})^{0}$. Thus $A \simeq\left(\mathbf{R}_{+}^{\times}\right)^{l}$, where $l=\operatorname{rank}_{\mathbf{Q}}(G)$.

Now let $e \in X$ be the base-point corresponding to $K$. Let $\bar{X}$ be the Borel-Serre bordification of $X[\mathbf{2}]$. From the construction of $\bar{X}$ given in [2], it is easy to verify the following:

LEMMA 1. The closure $Z$ of Ae in $\bar{X}$ is homeomorphic to a ball of dimension l. The boundary of $Z$ lies in $\partial \bar{X}$.

Now fix an orientation on $Z$.

LEMMA 2. The fundamental class of $\partial Z$ freely generates $H_{l-1}(\partial \bar{X}, \mathbf{Z})$ as a $\mathbf{Z}[U(\mathbf{Q})]$-module, where $U$ is the unipotent radical of a minimal $\mathbf{Q}$-parabolic subgroup $P$ of $G$ containing $T$.

The proof of this lemma is essentially contained in $\S 8$ of [2]. The central object is the Tits building $B$ of the Q-group $G$. The homotopy equivalence $g_{x}: B \rightarrow \partial \bar{X}$ constructed in $[2,8.4 .3]$ maps one of the apartments of $B$ homeomorphically onto $\partial Z$, if we choose $x$ to be the identity coset $e$ in $X$. Call this distinguished apartment $A_{0}$. Then choosing an $(l-1)$-dimensional simplex $s$ of $A_{0}$ corresponds to the choice of $P$ in the lemma. Each apartment is homeomorphic to an $(l-1)$-sphere and $B$

Received by the editors November 21, 1984 and, in revised form, April 25, 1985.

1980 Mathematics Subject Classification. Primary 20H05, 20 J99.

${ }^{1}$ Partially supported by a grant from the NSF. 
is homotopy equivalent $[2,8.5 .2]$ to the wedge of all the apartments of $B$ that contain $s$. Hence $\partial \bar{X}$ is homotopy-equivalent to a wedge of spheres, and $[2,8.6 .8]$ implies that $H_{l-1}(\partial \bar{X}, \mathbf{Z})$ is a free $\mathbf{Z}[U(\mathbf{Q})]$-module of rank 1 , generated by the fundamental class of $g_{e}\left(A_{0}\right)=\partial Z$.

We shall use the notation $[I]$ for the fundamental class of $\partial Z$ in $H_{l-1}(\partial \bar{X}, \mathbf{Z})$, where $I$ stands for the identity in $G(\mathbf{Q})$. More generally, if $M$ is any element of $G(\mathbf{Q})$, set $[M]$ to be $M \cdot[I]$, i.e. the fundamental class of $\partial(M Z)$. We call $[M]$ a universal minimal modular symbol. ("Minimal" refers to the fact that it is associated with a minimal Q-parabolic subgroup of $G$.)

Now let $\pi$ denote the canonical projection of $\bar{X}$ onto $\Gamma \backslash \bar{X}$. From now on, assume $l \geq 1$. Since $\bar{X}$ is contractible, $H_{l-1}(\partial \bar{X}, \mathbf{Z})$ is isomorphic to $H_{l}(X, \partial \bar{X}, \mathbf{Z})$. Now define $[M]_{\Gamma}$, for any $M$ in $G(\mathbf{Q})$, to be the image of $[M]$ in $H_{l}(\Gamma \backslash \bar{X}, \partial(\Gamma \backslash \bar{X}), \mathbf{Z})$ under the composition

$$
H_{l-1}(\partial \bar{X}, \mathbf{Z}) \stackrel{\sim}{\rightarrow} H_{l}(\bar{X}, \partial \bar{X}, \mathbf{Z}) \stackrel{\pi_{*}}{\rightarrow} H_{l}(\Gamma \backslash \bar{X}, \partial, \mathbf{Z}) .
$$

It follows from Lemma 2.7 of [4] that $\pi(M(A e))$ is a submanifold (with boundary) of $\Gamma \backslash \bar{X}$. Then $[M]_{\Gamma}$ is its fundamental class.

THEOREM. If $\Gamma$ is torsion-free, the modular symbols $[M]_{\Gamma}$ as $M$ runs through $U(\mathbf{Q})$, or a fortiori through $G(\mathbf{Q})$, generate $H_{l}(\Gamma \backslash \bar{X}, \partial, \mathbf{Z})$.

PROOF. This follows from the fact that $\pi_{*}$ is surjective. Although the proof of this fact is done in a special case in $[\mathbf{1}]$, the argument carries over verbatim.

REMARK. By Poincaré duality, $H_{l}(\Gamma \backslash \bar{X}, \partial, \mathbf{Z})$ is naturally isomorphic to $H^{N}(\Gamma \backslash \bar{X}, \mathbf{Z})$ when $\Gamma$ is torson-free, so we may take the promised set $S$ to be the set of currents corresponding to the modular symbols $[M]_{\Gamma}, M$ in $U(\mathbf{Q})$.

If $\Gamma$ is not torsion-free, the theorem and remark remain true if $\mathbf{Z}$-coefficients are replaced by Q-coefficients (cf. remark, p. 246 of $[\mathbf{1}]$ ).

EXAMPLE. If $F$ is any finite extension of $\mathbf{Q}$, let $G$ be the $\mathbf{Q}$-group such that $G(\mathbf{Q})=\mathrm{SL}(n, F), n \geq 2$. Then

$$
G(\mathbf{R})=\mathrm{SL}\left(N, F \otimes_{\mathbf{Q}} \mathbf{R}\right) .
$$

View $\mathbf{R}$ as embedded in the natural way in $F \otimes \mathbf{R}$. Then $A$ may be taken to be the diagonal matrices in $G(\mathbf{R})$ with entries in $\mathbf{R}_{+}^{\times}$, and $l=n-1$. In case the ring of integers in $F$ is Euclidean, the algorithm in $\S 4$ of $[\mathbf{1}]$ shows how to reduce $S$ to a finite spanning set. But otherwise, a general algorithm for this problem is unknown and would be of great interest.

REMARKS. (1) Nonminimal modular symbols may be defined in other dimensions, as in [4]. For these symbols, no such assertion that they generate the cohomology in their dimension is known to be true, nor is any counterexample known to me.

(2) The set $S=\left\{[M]_{\Gamma}: M \in U(\mathbf{Q})\right\}$ depends upon the choices of $T, K$ and $U$. We may enlarge $S$ to obtain a set which does not depend on these choices, namely $\left\{[M]_{\Gamma}: M \in G(\mathbf{Q})\right\}$. 


\section{REFERENCES}

1. A. Ash and L. Rudolph, The modular symbol and continued fractions in higher dimensions, Invent. Math. 55 (1979), 241-250.

2. A. Borel and J.-P. Serre, Corners and arithmetic groups, Comment Math. Helv. 48 (1973), 436-491.

3. A. Borel, Introduction aux groupes arithmétiques, Hermann, Paris, 1969.

4. A. Ash, Non-square-integrable cohomology of arithmetic groups, Duke Math. J. 47 (1980), 435-449.

Department of Mathematics, Ohio State University, Columbus, Ohio 43210 